\title{
Synthetic and Isolation Studies Related to the Marine Natural
}

\section{Products (+)-Elisabethadione and (+)-Elisabethamine}

\author{
Xing Dai ${ }^{a}$, Zhongliang Wan $^{b}$, Russell G. Kerr ${ }^{b, c}$, and Huw M. L. Davies ${ }^{a, *}$
}

${ }^{a}$ Department of Chemistry, University at Buffalo, The State University of New York, Buffalo, New York, 14260-3000, USA

${ }^{\mathrm{b}}$ Department of Chemistry and Biochemistry, Center of Excellence in Biomedical and Marine Biotechnology, Florida Atlantic University, Boca Raton, Florida, 33431-0331, USA

${ }^{c}$ Department of Chemistry, University of Prince Edward Island, Charlottetown, PEI, C1A-4P3, Canada

\section{Supporting Information}

\section{Contents}

Title of the paper, authors' name, address and contents.

${ }^{1}$ HNMR spectra of $\mathbf{1 6}$

${ }^{13}$ CNMR spectra of $\mathbf{1 6}$

${ }^{1}$ HNMR spectra of Synthetic $O$-methyl-elisabethadione (8)

${ }^{13}$ CNMR spectra of Synthetic O-methyl-elisabethadione (8)

${ }^{1}$ HNMR spectra of 19

${ }^{13}$ CNMR spectra of 19

${ }^{1}$ HNMR spectra of Synthetic $O$-methyl-nor-elisabethadione (9)

${ }^{13}$ CNMR spectra of Synthetic O-methyl-nor-elisabethadione (9)

${ }^{1}$ HNMR spectra of $\mathbf{2 0}$

${ }^{13}$ CNMR spectra of 20

${ }^{1}$ HNMR spectra of Natural $O$-methyl-elisabethadione (8)

${ }^{13} \mathrm{CNMR}$ spectra of Natural $O$-methyl-elisabethadione (8)

\section{Page Number}

S1

S3

S4

S5

S6

S7

S8

S9

S10

S11

S12

S13

S14 
HRMS of Natural O-methyl-elisabethadione (8)

S15

${ }^{1}$ HNMR spectra of Natural O-methyl-nor-elisabethadione (9)

S16

${ }^{13}$ CNMR spectra of Natural O-methyl-nor-elisabethadione (9)

S17

HRMS of Natural O-methyl-nor-elisabethadione (9)

S18 


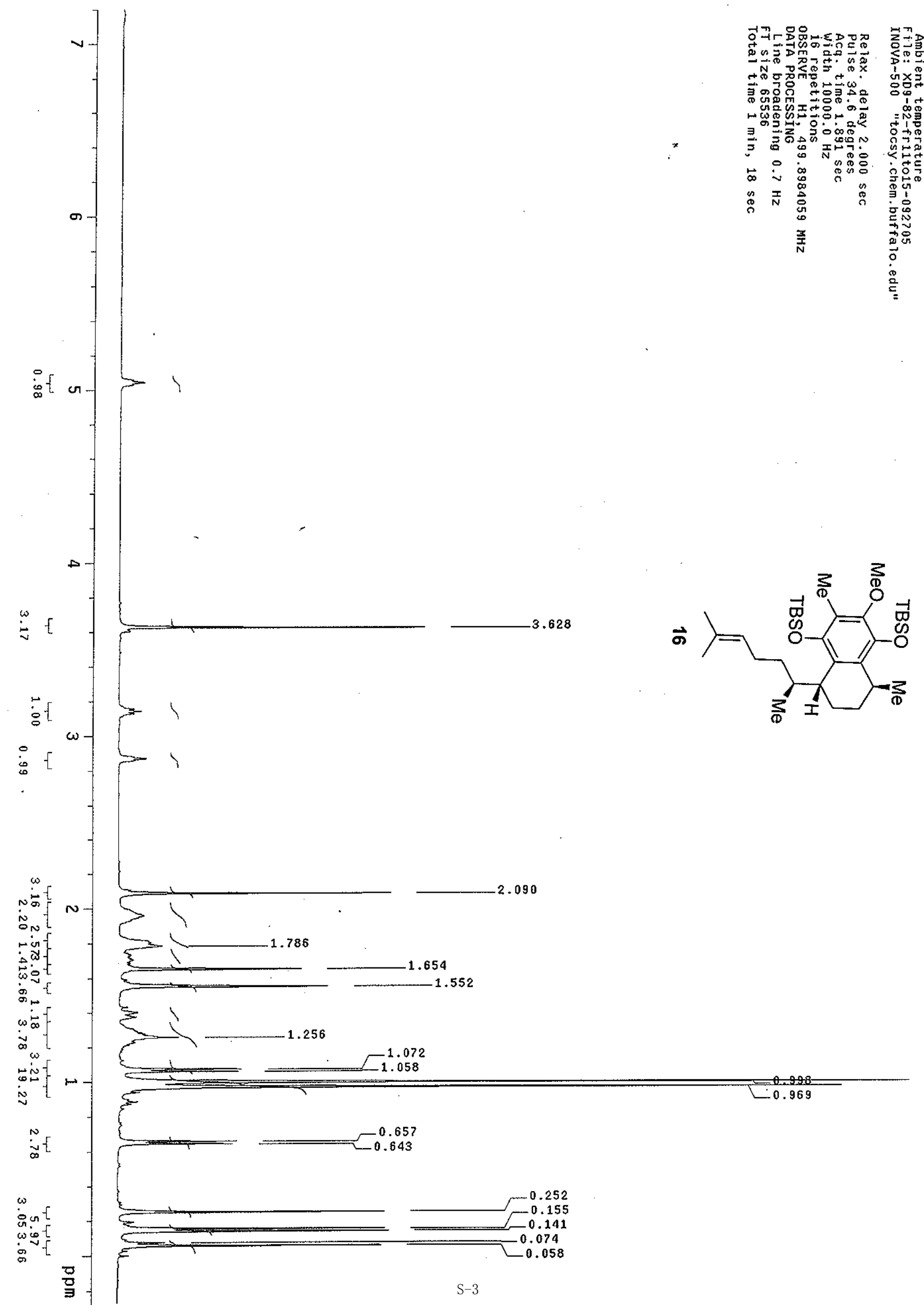




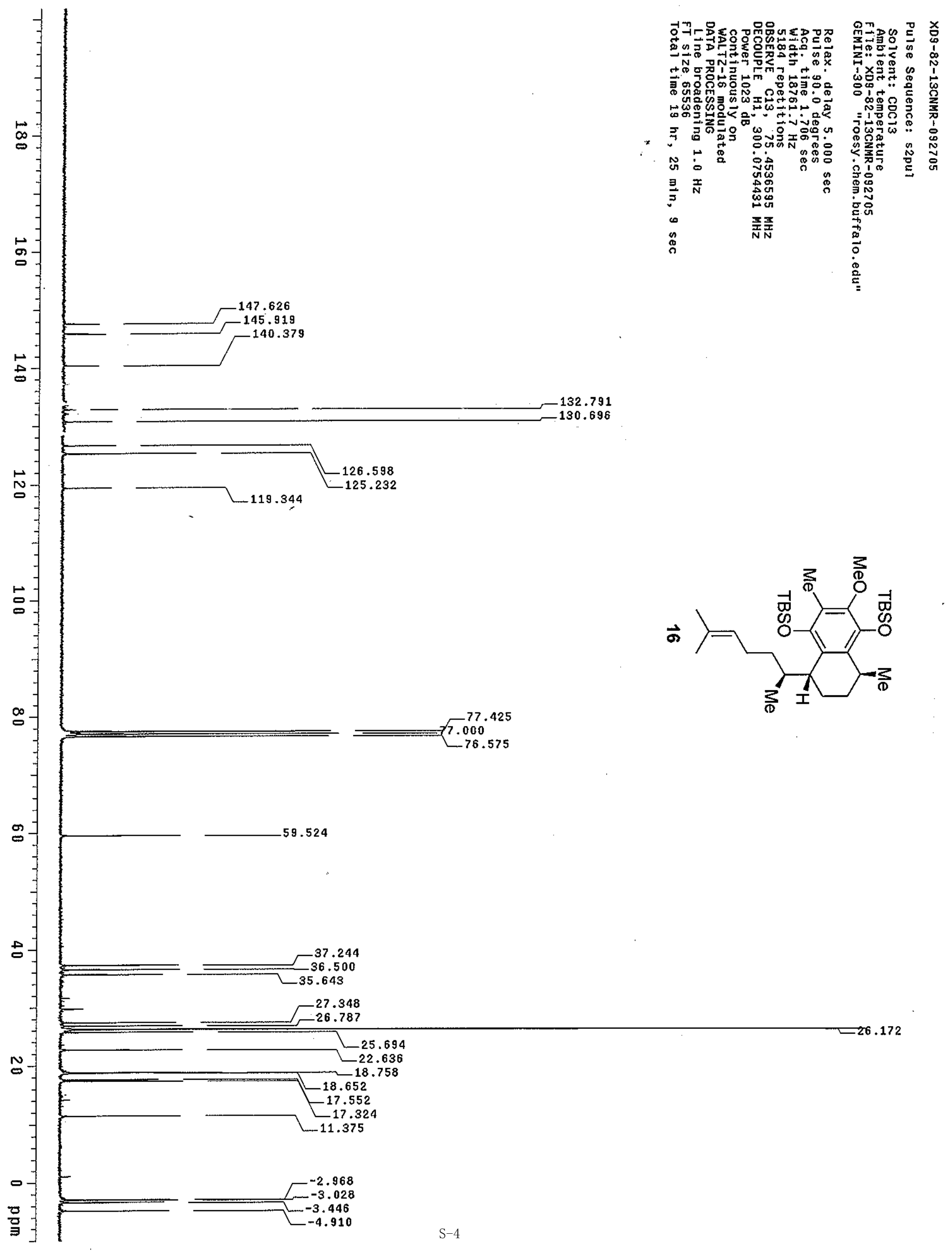




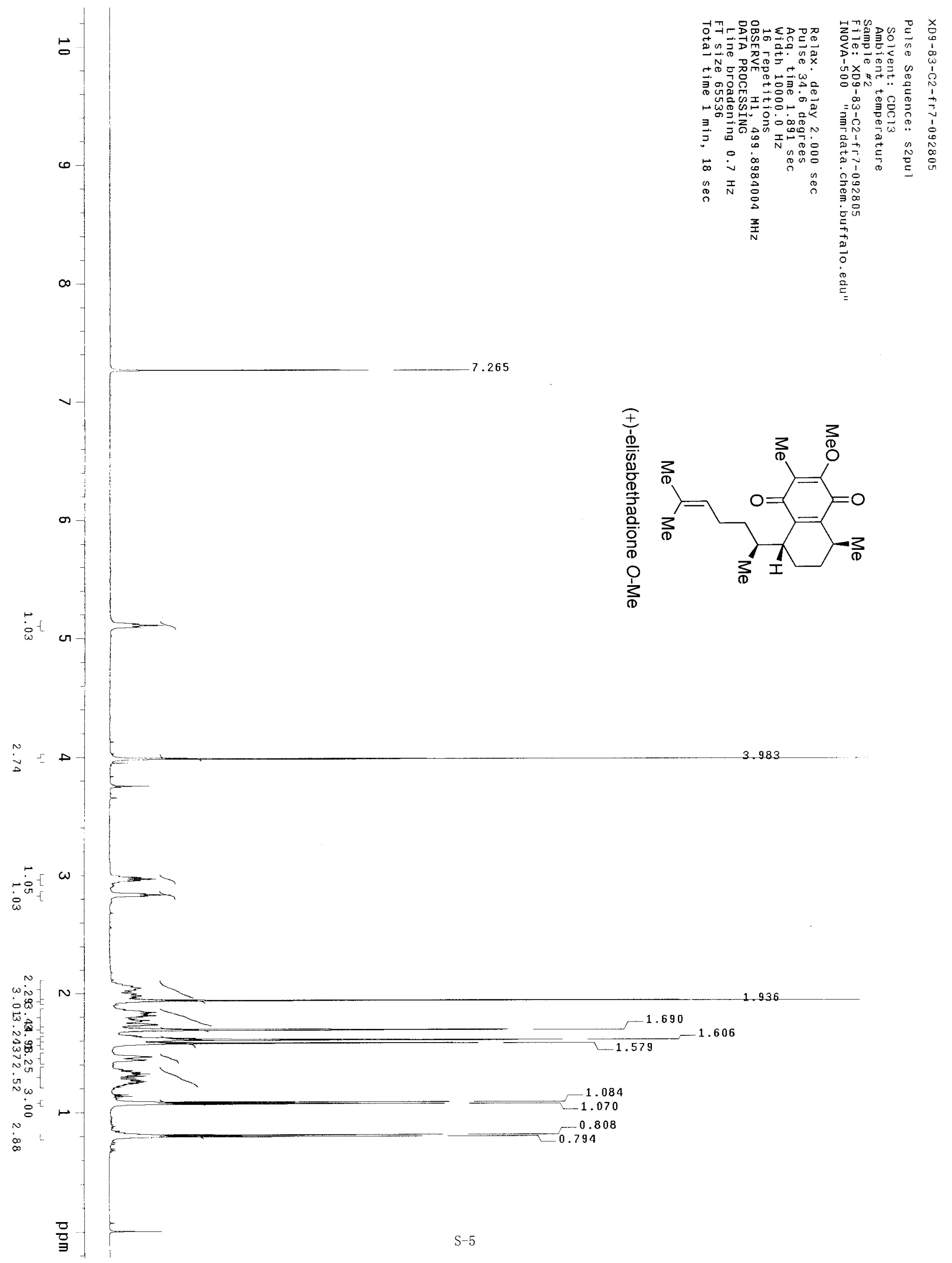



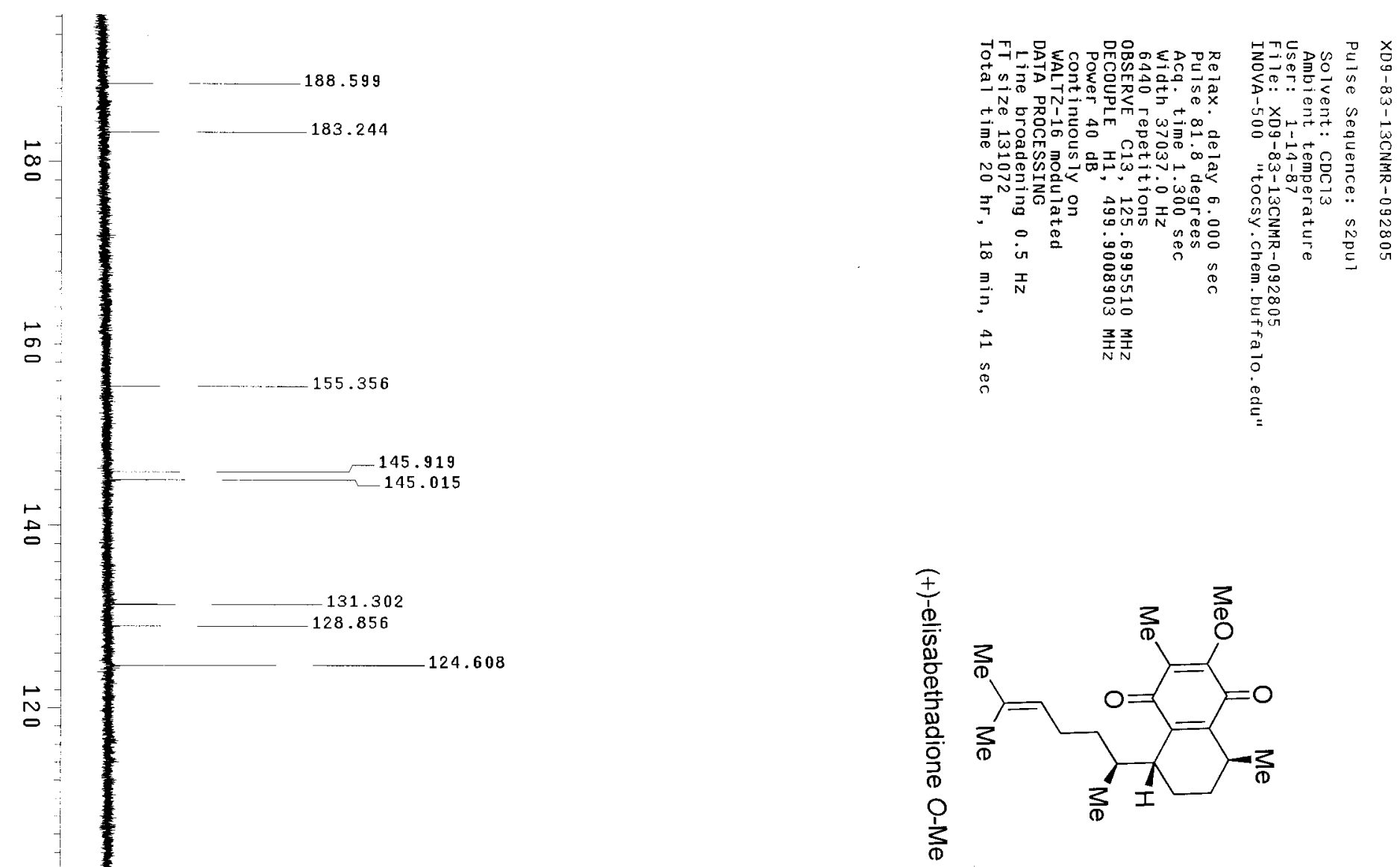

뭉

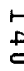

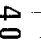

131.302

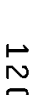

124.608

N

$-145.015$

$\stackrel{\infty}{\infty}$

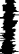

77.252

$-76.74$

욤

$-60.797$

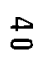

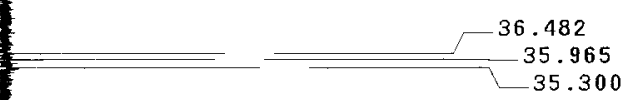

35.300

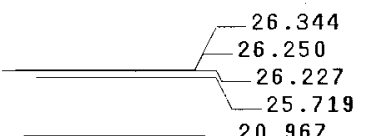

N

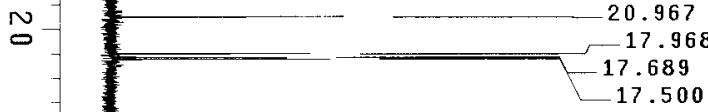

8.873

憘 


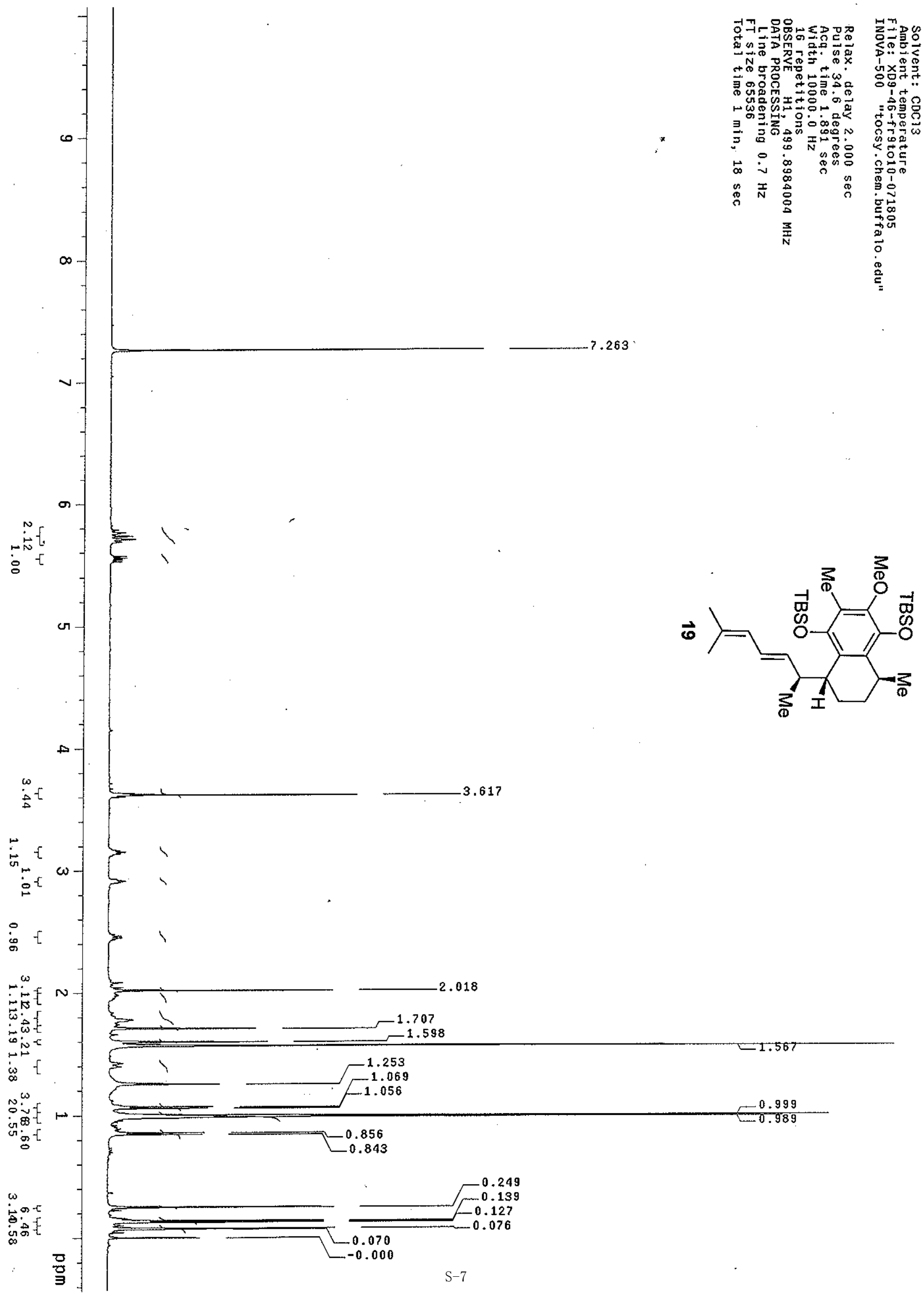




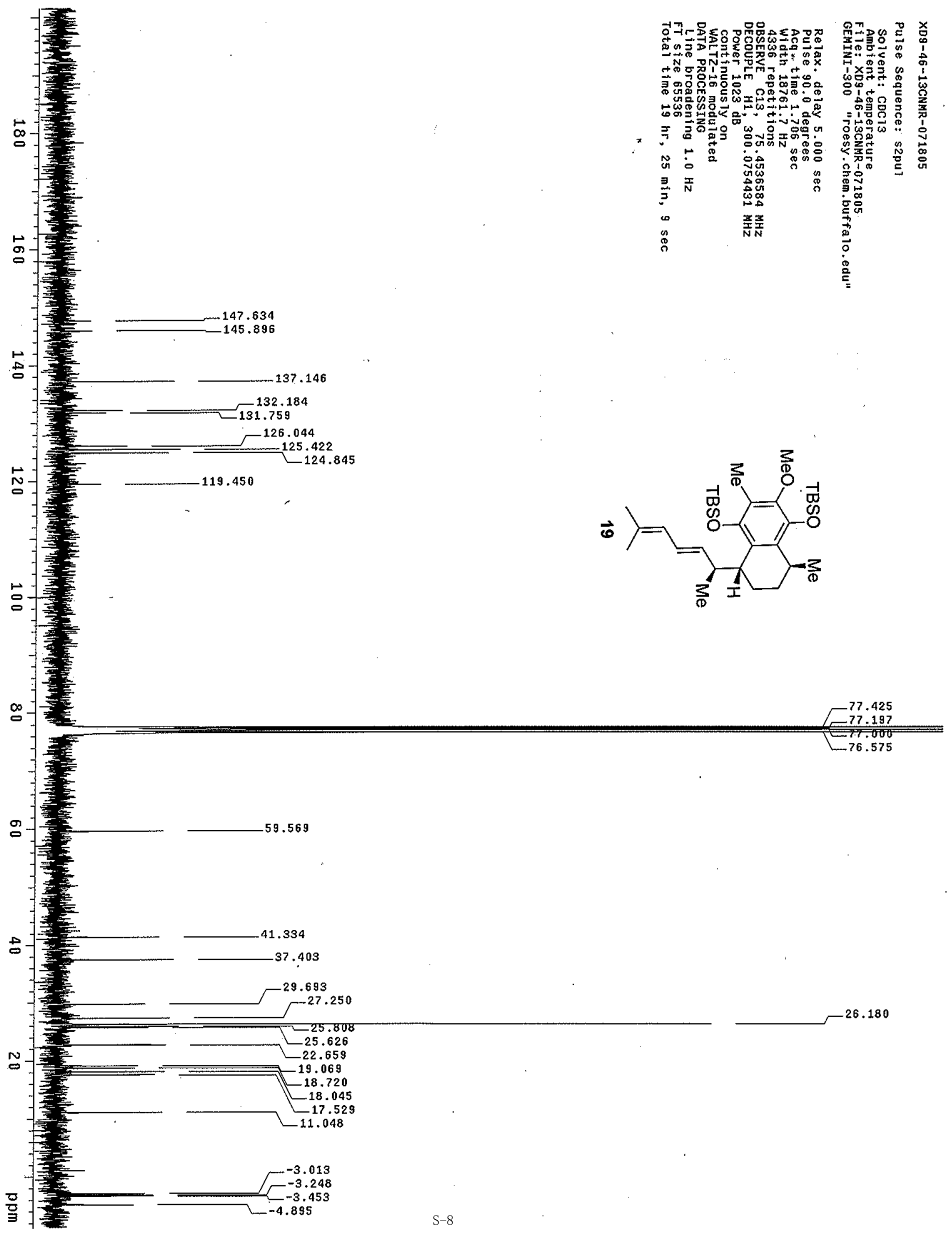




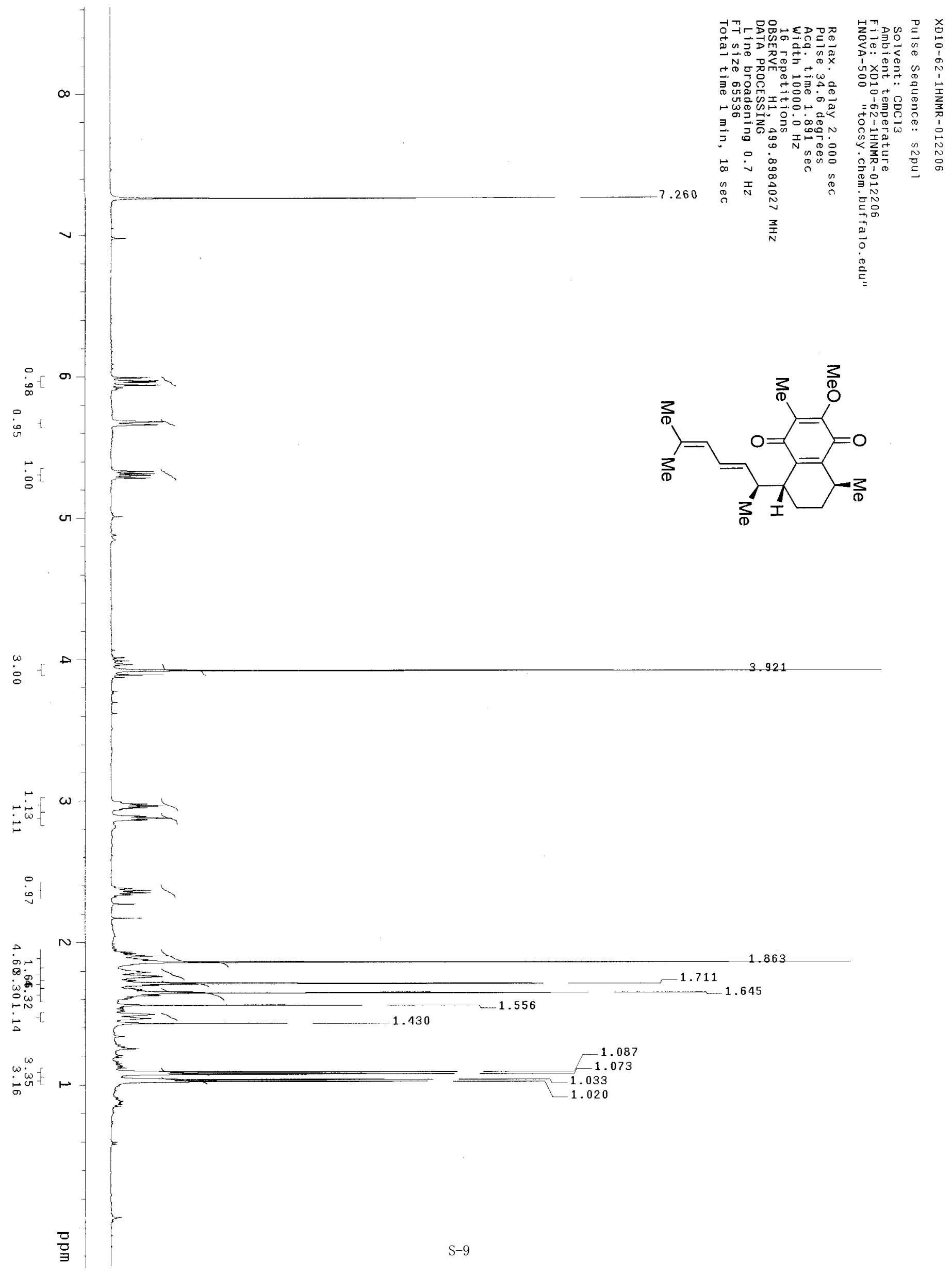



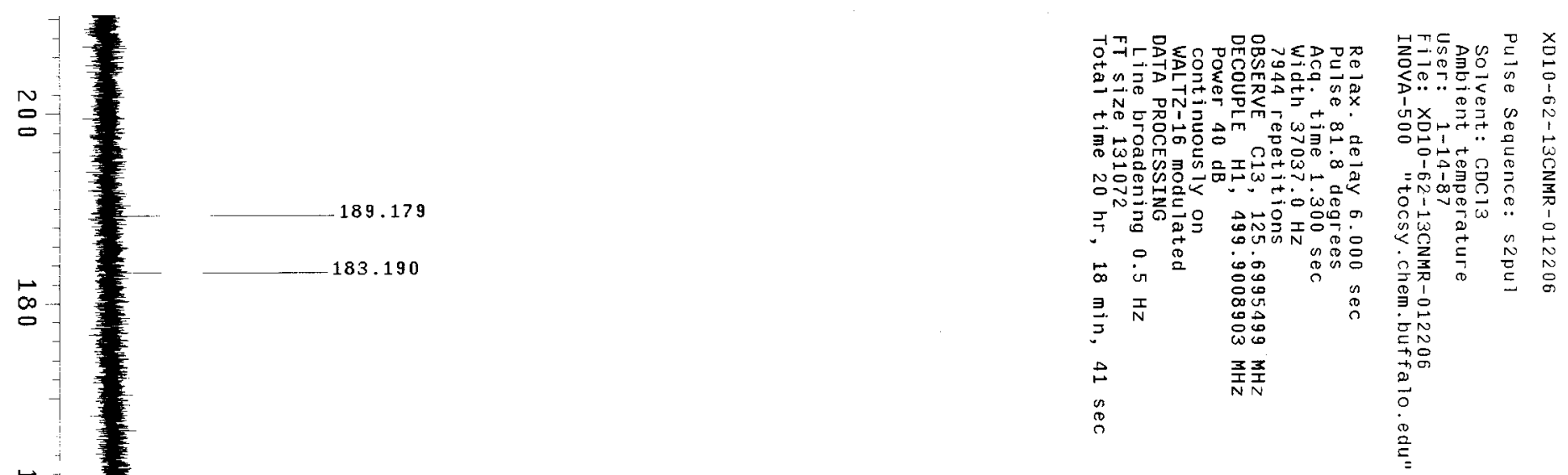

145.914

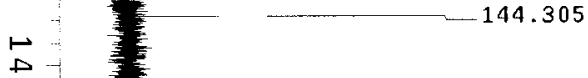

137.066

133.460 $-129.396$

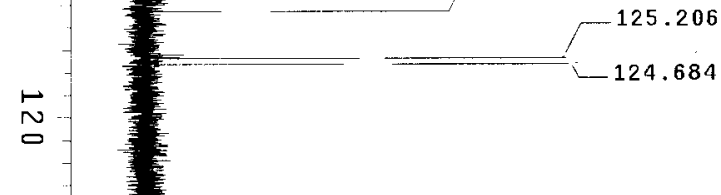

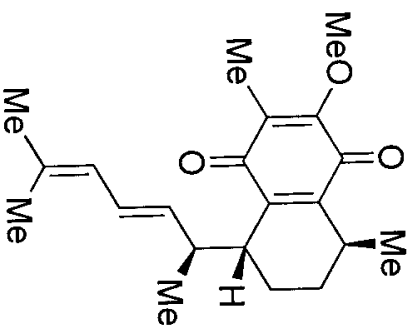

$\infty$

몽

154.960

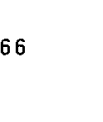




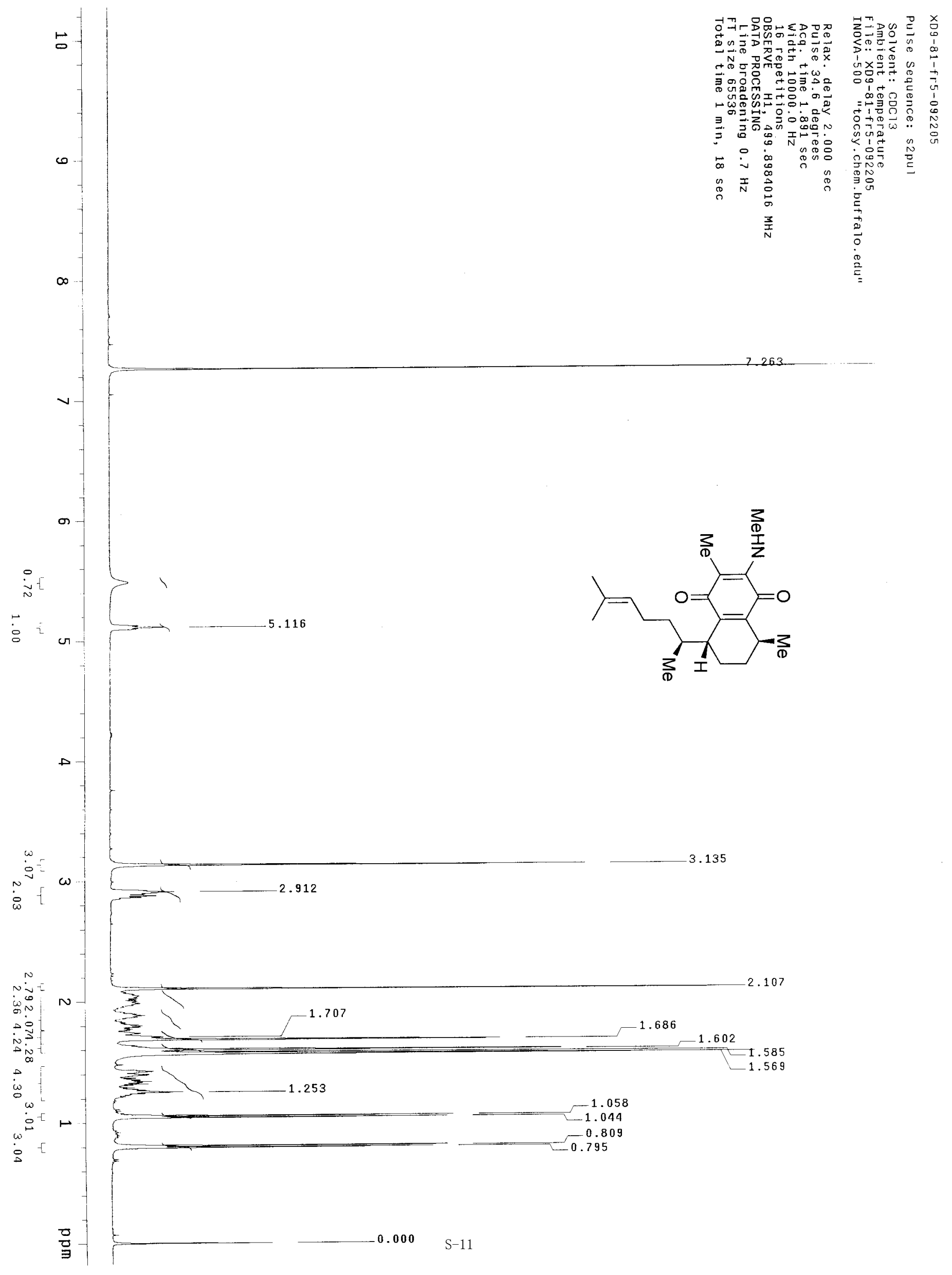




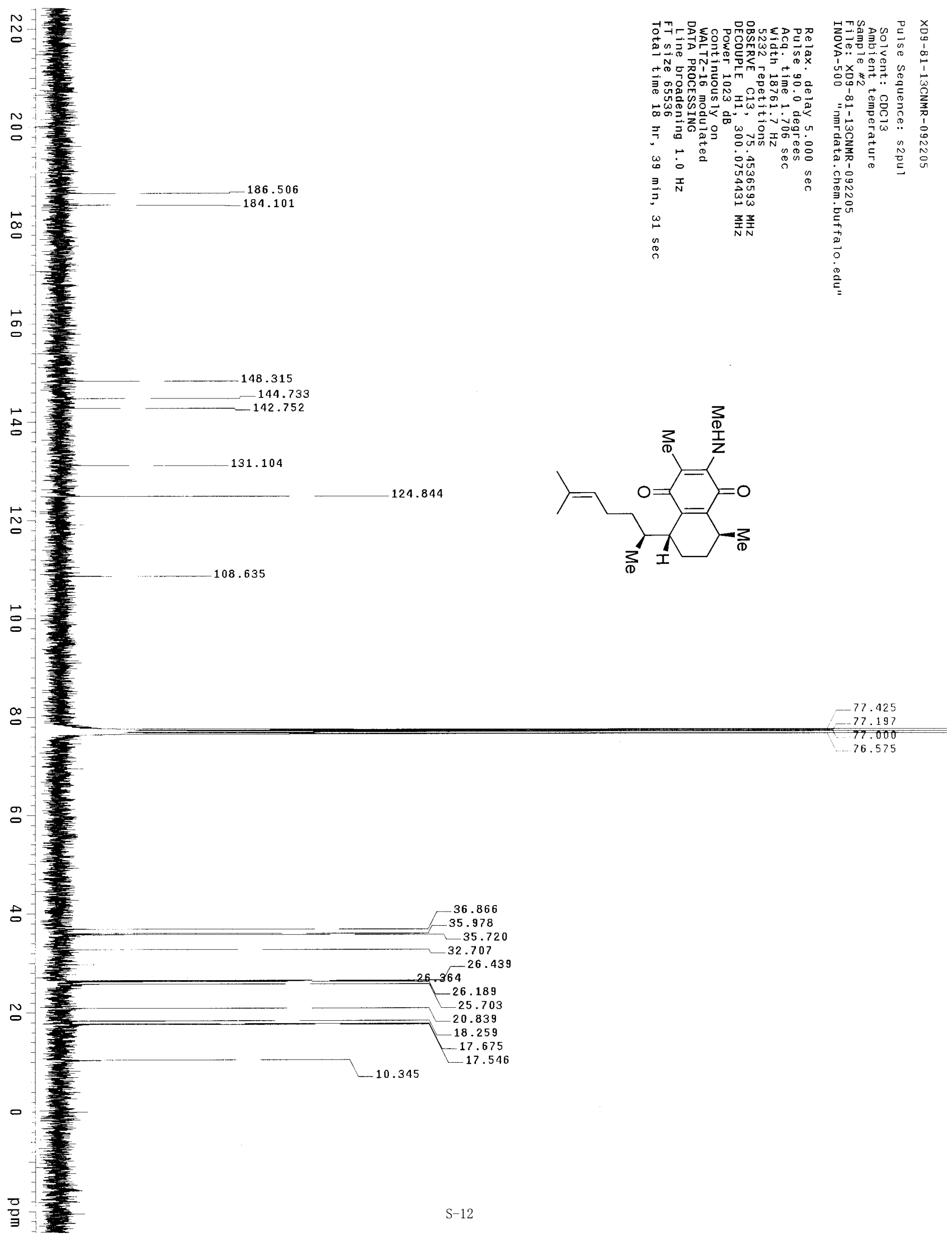



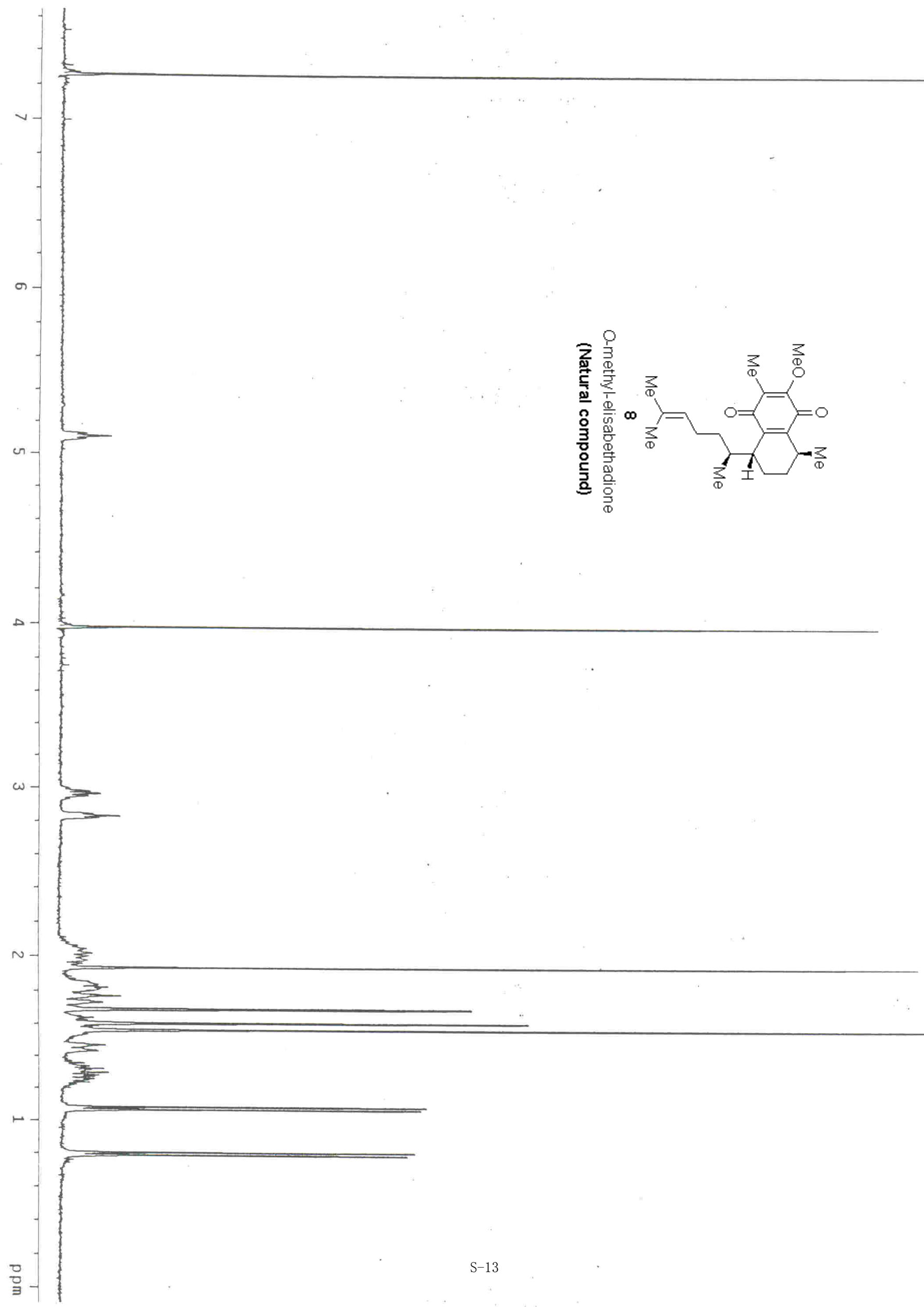


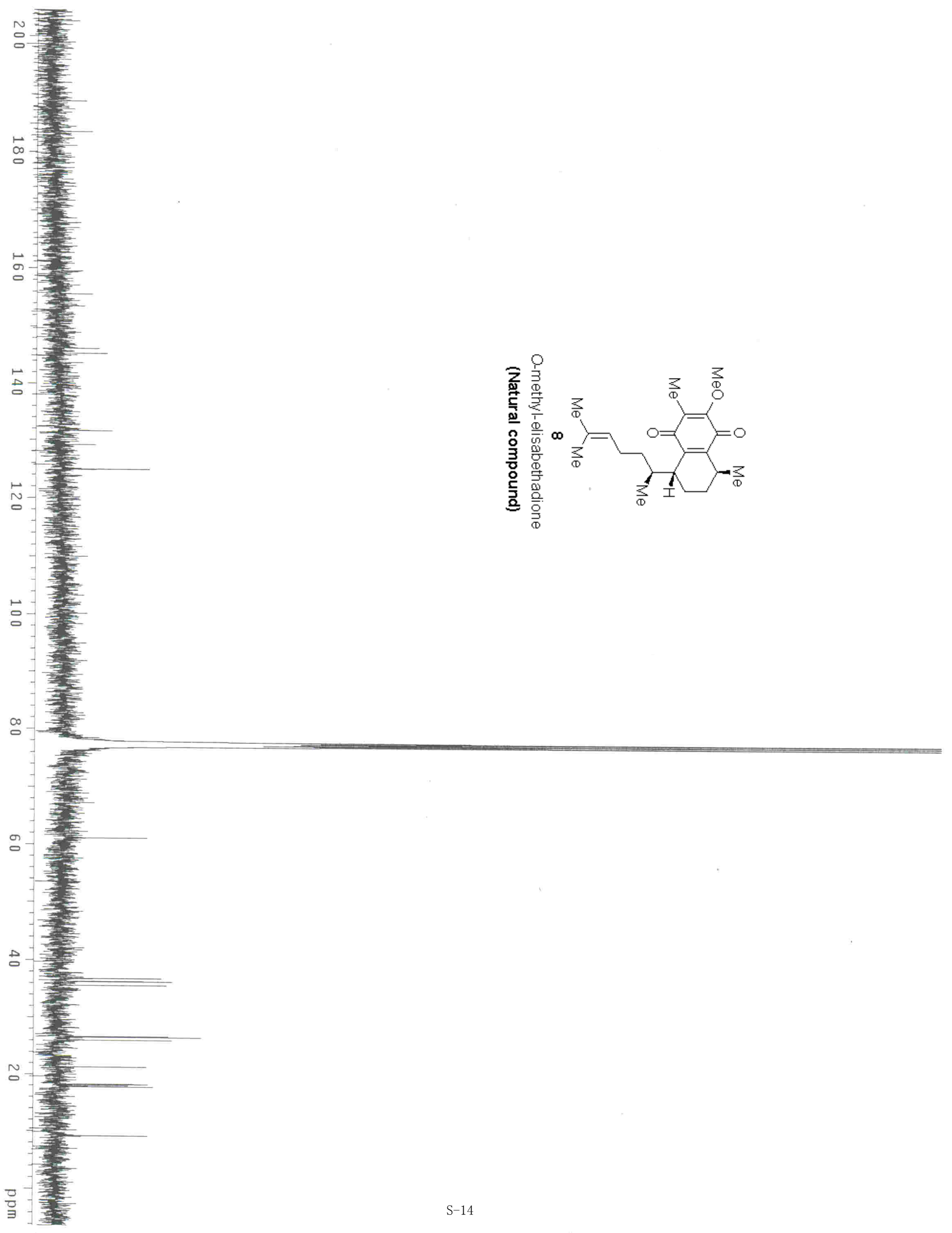




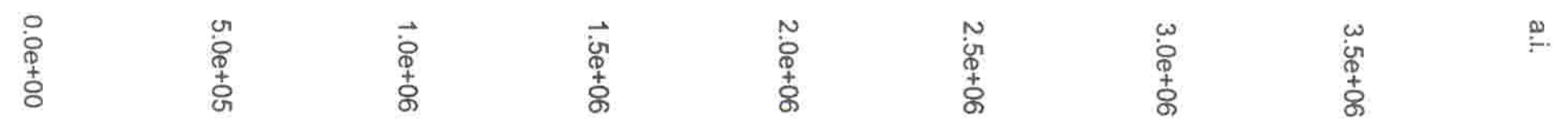
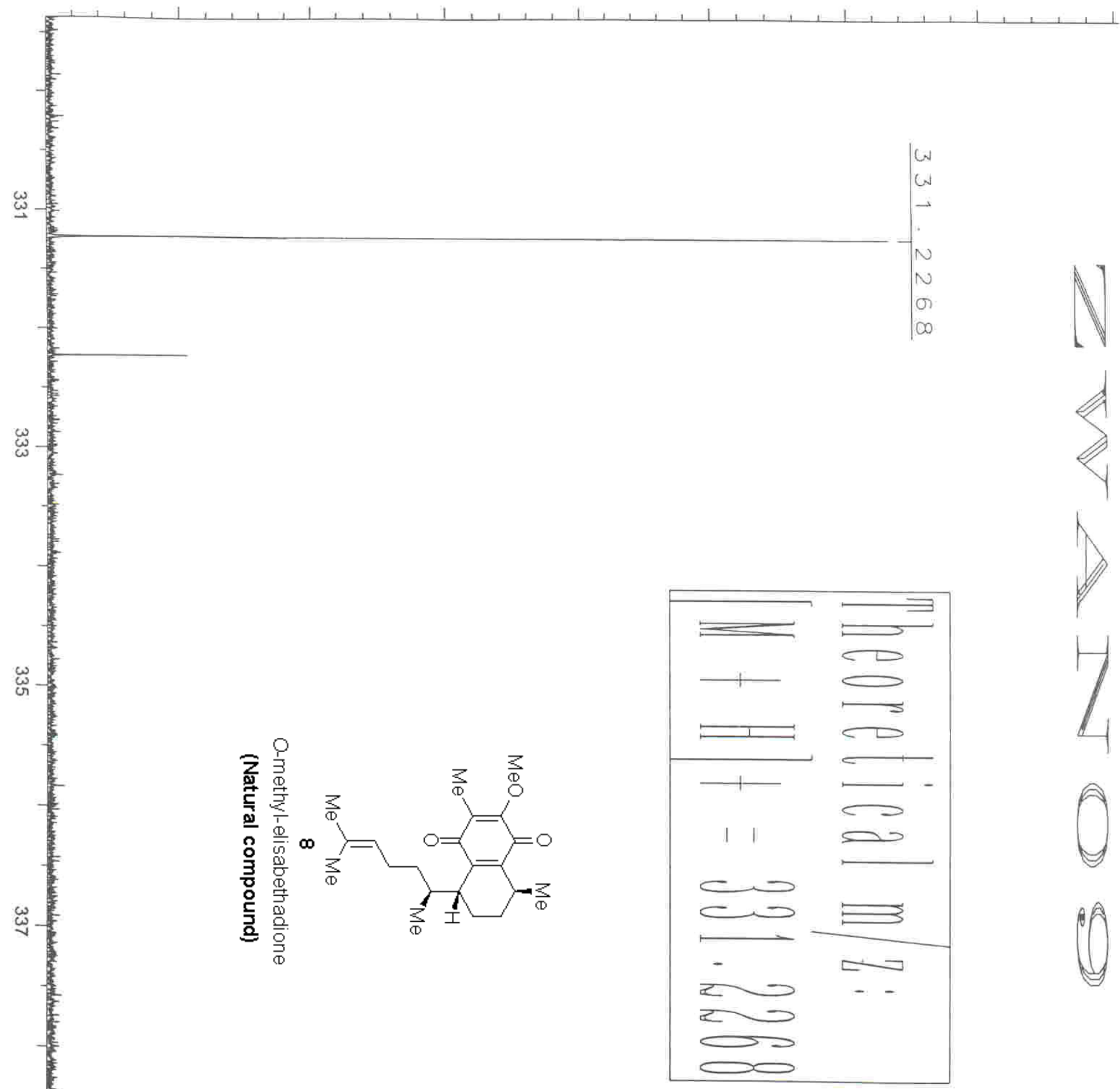


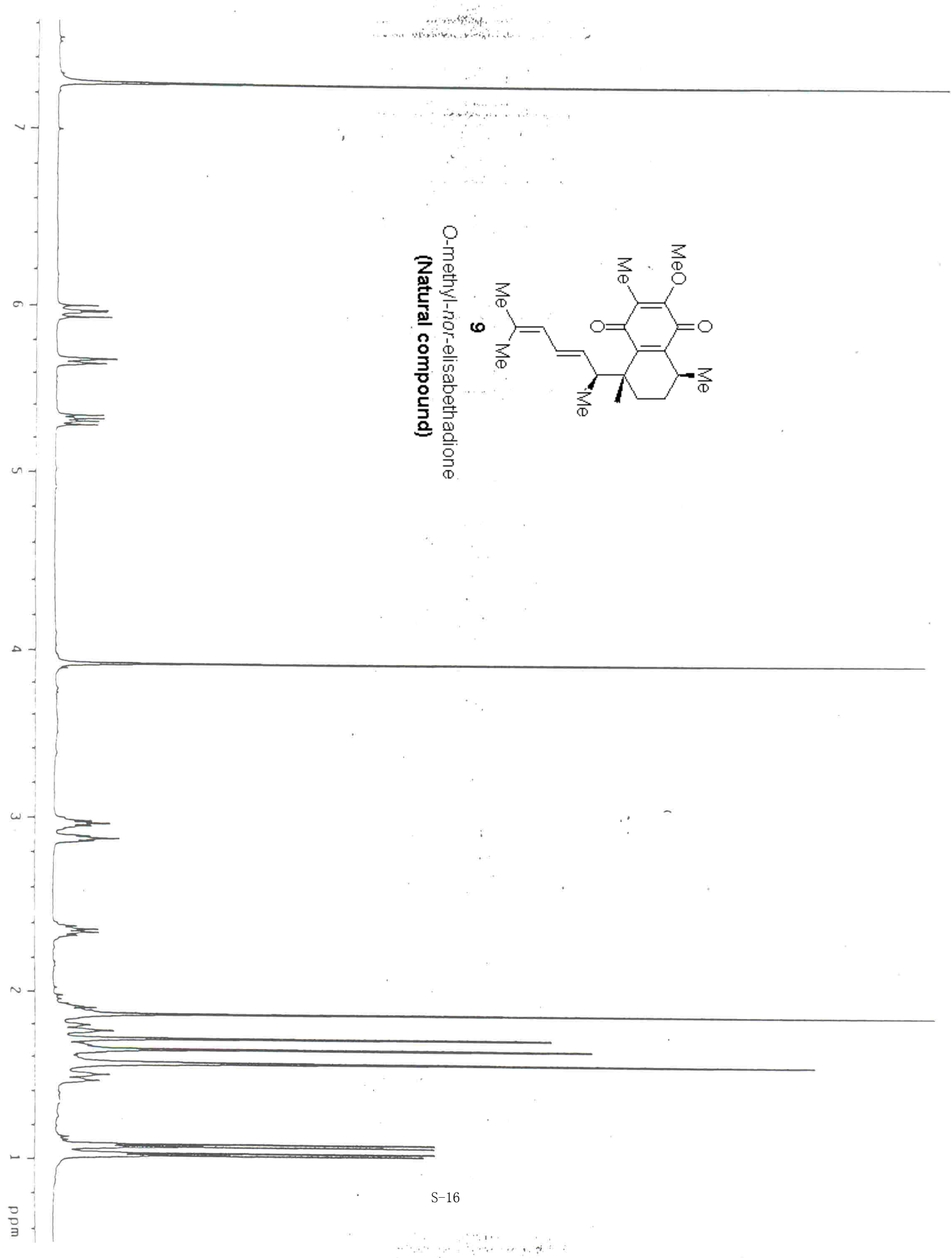




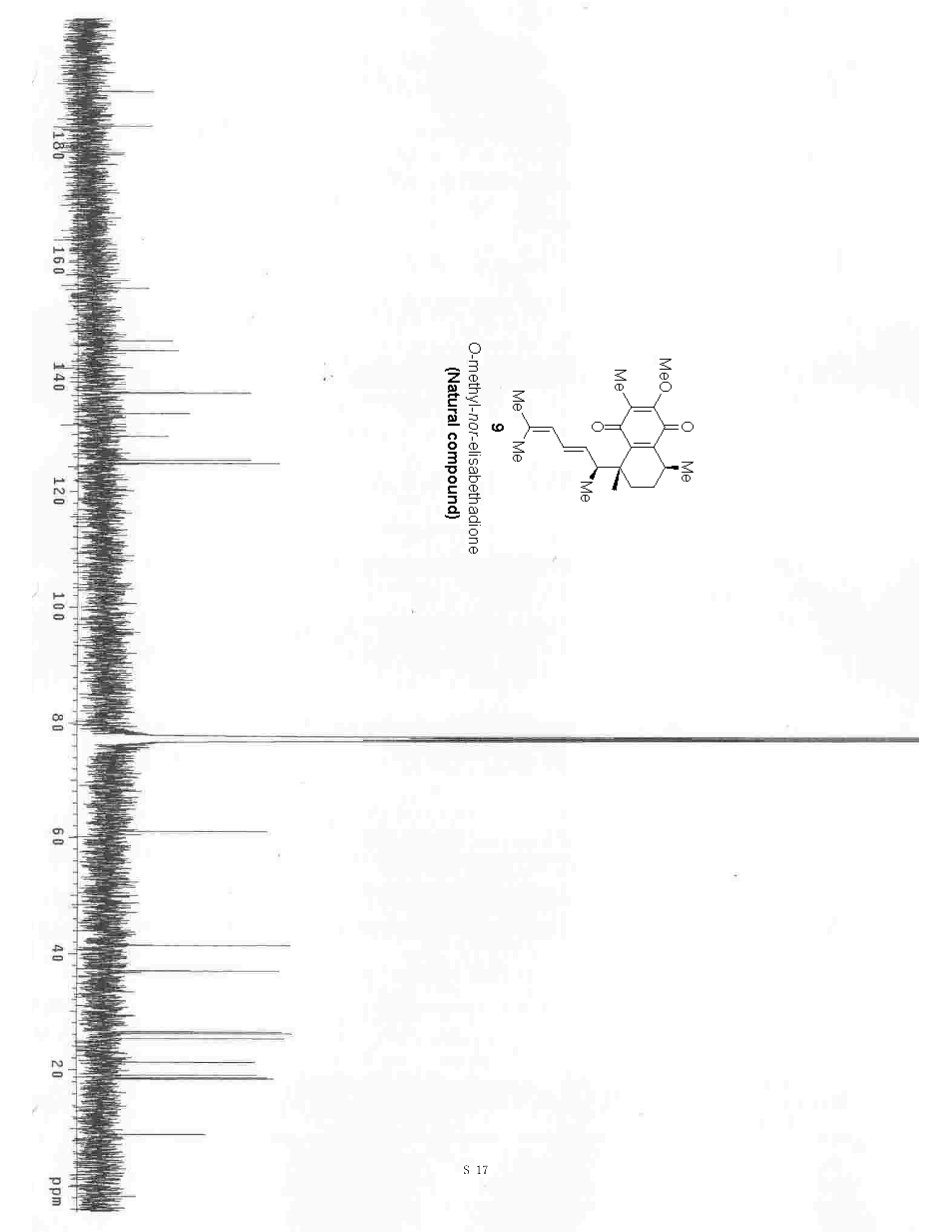




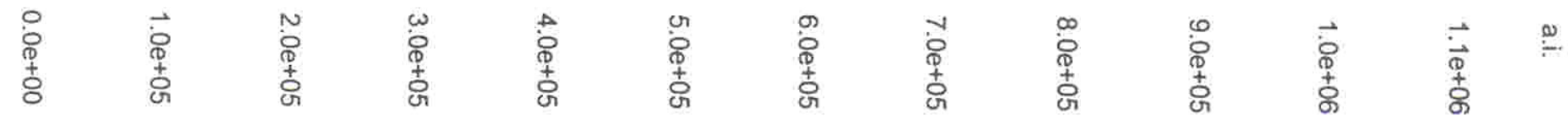
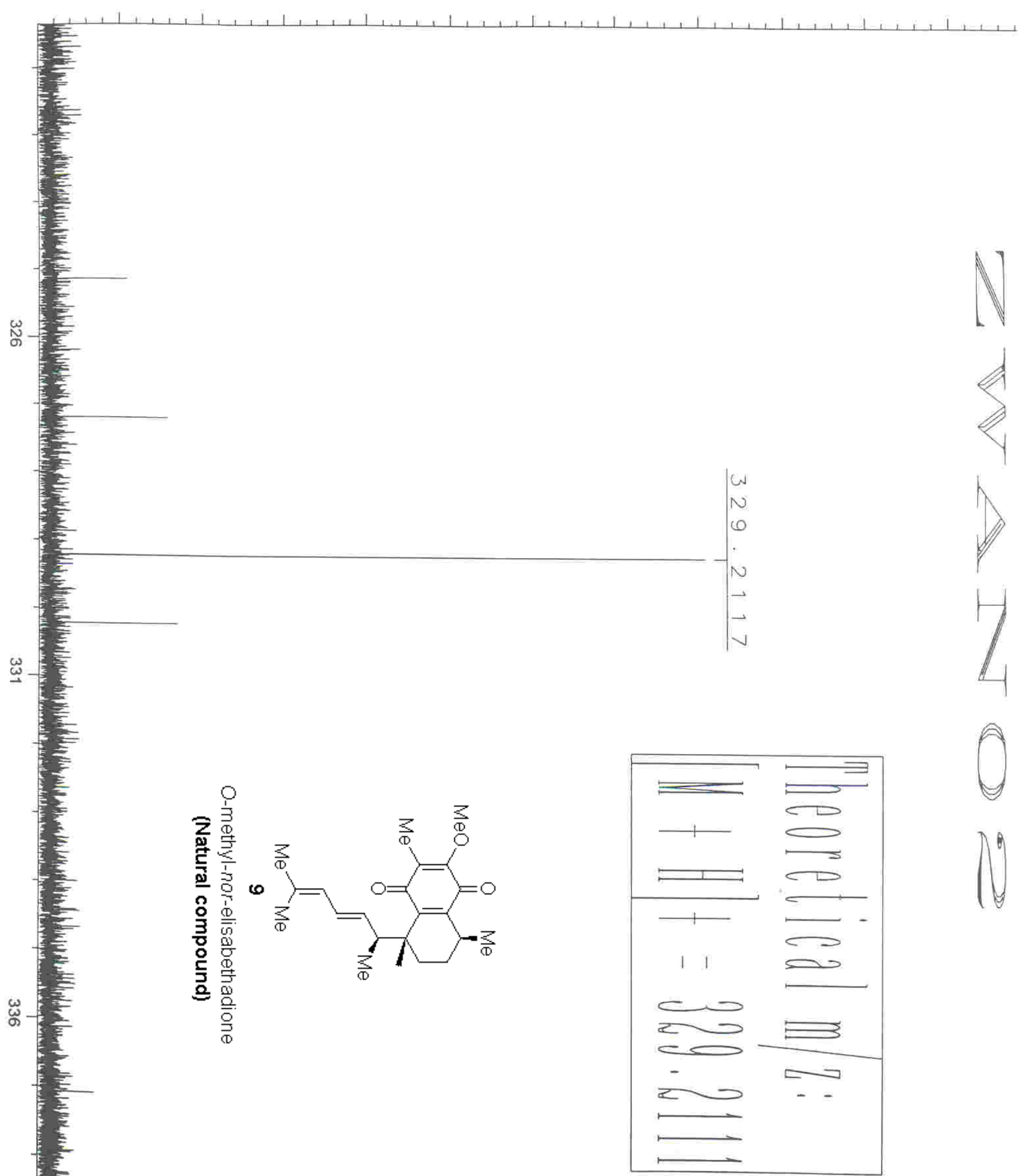